\title{
BanLec-eGFP Chimera as a Tool for Evaluation of Lectin Binding to High-Mannose Glycans on Microorganisms
}

\author{
Zorana Lopandić ${ }^{1}$, Luka Dragačević ${ }^{2}$, Dragan Popović ${ }^{3}$, Uros Andjelković $^{3,4}$, Rajna Minić ${ }^{2}$ and \\ Marija Gavrović-Jankulović ${ }^{1, * \mathbb{B}}$ \\ 1 Department of Biochemistry, Faculty of Chemistry, University of Belgrade, 11000 Belgrade, Serbia; \\ lopandic93@gmail.com \\ 2 Institute of Virology, Vaccines and Sera, 11152 Belgrade, Serbia; lukadragacevic@gmail.com (L.D.); \\ minicrajna@gmail.com (R.M.) \\ 3 Institute of Chemistry, Technology and Metallurgy, National Institute of the Republic of Serbia, University of \\ Belgrade, 11000 Belgrade, Serbia; dpopovic@chem.bg.ac.rs (D.P.); uros@chem.bg.ac.rs (U.A.) \\ 4 Department of Biotechnology, University of Rijeka, 5100 Rijeka, Croatia \\ * Correspondence: mgavrov@chem.bg.ac.rs; Tel.: +381-11-3336-661
}

check for updates

Citation: Lopandić, Z.; Dragačević, L.; Popović, D.; Andjelković, U.; Minić, R.; Gavrović-Jankulović, M. BanLec-eGFP Chimera as a Tool for Evaluation of Lectin Binding to High-Mannose Glycans on Microorganisms. Biomolecules 2021, 11, 180. https://doi.org/10.3390/ biom 11020180

Academic Editor: Andrey

P. Anisimov

Received: 18 November 2020

Accepted: 22 January 2021

Published: 28 January 2021

Publisher's Note: MDPI stays neutral with regard to jurisdictional claims in published maps and institutional affiliations.

Copyright: (c) 2021 by the authors. Licensee MDPI, Basel, Switzerland. This article is an open access article distributed under the terms and conditions of the Creative Commons Attribution (CC BY) license (https:// creativecommons.org/licenses/by/ $4.0 /)$.

\begin{abstract}
Fluorescently labeled lectins are useful tools for in vivo and in vitro studies of the structure and function of tissues and various pathogens such as viruses, bacteria, and fungi. For the evaluation of high-mannose glycans present on various glycoproteins, a three-dimensional (3D) model of the chimera was designed from the crystal structures of recombinant banana lectin (BanLec, Protein Data Bank entry (PDB): 5EXG) and an enhanced green fluorescent protein (eGFP, PDB 4EUL) by applying molecular modeling and molecular mechanics and expressed in Escherichia coli. BanLeceGFP, produced as a soluble cytosolic protein of about $42 \mathrm{kDa}$, revealed $\beta$-sheets $(41 \%)$ as the predominant secondary structures, with the emission peak maximum detected at $509 \mathrm{~nm}$ (excitation wavelength $488 \mathrm{~nm}$ ). More than $65 \%$ of the primary structure was confirmed by mass spectrometry. Competitive BanLec-eGFP binding to high mannose glycans of the influenza vaccine (Vaxigrip ${ }^{\circledR}$ ) was shown in a fluorescence-linked lectin sorbent assay (FLLSA) with monosaccharides (mannose and glucose) and wild type BanLec and H84T BanLec mutant. BanLec-eGFP exhibited binding to mannose residues on different strains of Salmonella in flow cytometry, with especially pronounced binding to a Salmonella Typhi clinical isolate. BanLec-eGFP can be a useful tool for screening high-mannose glycosylation sites on different microorganisms.
\end{abstract}

Keywords: banana lectin; eGFP; fluorescence; viral glycoproteins; influenza vaccine; florescencelinked lectin sorbent assay; Salmonella strains

\section{Introduction}

Lectins are carbohydrate-binding proteins involved in many biological processes like recognition and binding of glycans, host-pathogen interactions, cell targeting, cell-cell communication, induction of apoptosis, cancer metastasis, and differentiation [1,2]. Many different lectins are known and their glycan-binding specificities characterized (consortium for functional glycomics at http:/ / www.functionalglycomics.org/). Antimicrobial activities of lectins toward viruses, fungi, and bacteria have been reported [3], with plant lectins as the well-characterized group.

The first report on the isolation of banana lectin (BanLec) was from Musa paradisiac [4]. It belongs to the jacalin-related superfamily (mJRL) of lectins, which recognizes and binds to high mannose glycans [5]. It is homodimeric in solution, with a molecular mass of about $26 \mathrm{kDa}$. Each subunit consists of twelve $\beta$-strands arranged in a $\beta$-Prism-I fold $[6,7]$. BanLec is an IgG4-inducing antigen [8] and a potent inhibitor of human immunodeficiency virus (HIV) replication $[9,10]$. BanLec binds to the glycosylated proteins in the viral envelope and inhibits its cellular entry by blocking glycan-mediated interactions with the 
CD4 receptor and CCR5/CCR4 co-receptors on host cells [10]. Because of its ability to induce T cell proliferation, BanLec has also been recognized as a potential immunomodulatory molecule [11-13]. It was shown that mutation of the amino acid at position 84 from histidine to threonine minimizes the wild-type banana lectin's mitogenicity, without compromising antiviral activity against viruses that have high-mannose-type $\mathrm{N}$-glycans on their surfaces [14].

Fluorescently labeled lectins are useful tools for both in vivo and in vitro study of the structure and function of tissues [15-17], pathological processes, including cancer [18-20], as well as those initiated with pathogens such as viruses, bacteria, and fungi [21].

Lectins can be fluorescently labeled using a chemical approach. However, chemical labeling can change the secondary and tertiary structure of lectins and consequently impair their interaction with glycans [22]. The binding constant between lectins and glycans is usually low, and every change of lectin structure can introduce a change of binding site and potentially affect the binding constant.

Green fluorescent protein (GFP) is a ready-made fluorescent polypeptide that finds broad application in various assays and in vivo imaging. The most explored application is a genetic fusion with a protein of interest [23]. The fused protein preserves its function along with the acquired fluorescent property of the GFP expressing gene. For instance, a neurocan-GFP which specifically binds to hyaluronan was employed for revealing a detailed picture of hyaluronan tissue distribution [24], while eGFP-Nictaba, the cytoplas$\mathrm{mic} /$ nuclear tobacco lectin, can interact with high-mannose and complex N-glycans [25]. Fluorescently labeled Aspergillus oryzae lectin is useful tool for the detection of the $\alpha(1,6)$ fucose attached to the core N-glycan (core fucose) of glycoproteins known to play essential roles in various pathophysiological events [26].

In this study, we have designed in silico, produced, purified, and characterized recombinant BanLec-eGFP chimera. The application of BanLec-eGFP was tested with competitive florescence-linked lectin assay (FLLA) for studies of interactions between the lectin and high-mannose carbohydrates present on the trivalent inactivated A and $\mathrm{B}$ split-virion influenza vaccine; and with flow cytometry for the detection of high mannose content lipopolysaccharides (LPS) on the surface of bacteria.

\section{Materials and Methods}

\subsection{Strains and Plasmids}

Escherichia coli strain DH5 $\alpha$ (Invitrogen Thermo Fisher Scientific, Carlsbad, USA) was used as a host to propagate plasmids pUC57-eGFP (GenScript HK Limited, Hong Kong, China) and BanLec gene-containing pET-23b, pET-23b-BL. Bacteria were grown in LuriaBertani (LB) medium (10 g/L Tryptone (Torlak, Institute of Virology, Vaccines, and Sera, Belgrade, Serbia), $5 \mathrm{~g} / \mathrm{L} \mathrm{NaCl}$ (Betahem, Belgrade, Serbia), $5 \mathrm{~g} / \mathrm{L}$ yeast extract (Torlak), and $15 \mathrm{~g} / \mathrm{L}$ agar (Torlak)) supplemented with antibiotics when appropriate.

\subsection{Design, Cloning of eGFP in $p E T-23 b-B L$}

The three-dimensional (3D) model of the BanLec-eGFP chimeric structure is designed from the crystal structure of a recombinant banana lectin, Protein Data Bank (PDB) entry 5EXG [10] (chain A containing 141 amino acids) and the crystal structure of an enhanced green fluorescent protein, PDB entry 4EUL [27] (chain A containing 239 amino acids) by applying the molecular modeling and molecular mechanics to optimize the structure. All modifications in the sequence of BanLec-eGFP chimera relative to the used crystal structures, such as replacing the residues, removing the excessive, and adding the missing residues, were done in Discovery Studio 19.1.0. [28] The N-terminal part (MVS) and Cterminal fragment (GMDELYK) were modeled in a random coil conformation but ended up with a few turns and bends.

A glycine-serine-glutamate-phenylalanine (GSEF) linker was constructed between the BanLec and eGFP to prevent steric obstacles. The GSEF linker was modeled as a coil extension to the $\beta$-strand secondary structure of the C-terminus of eGFP in Discovery 
Studio 19.1.0 [28]. The computer-generated BanLec-eGFP chimeric design was obtained by connecting the three fragments and adding the hydrogen atoms, followed by the full structural minimization in NAMD 2.9 [29] with CHARMM22 force field [30] in implicit water model for 300 ps. The optimized resulting structure was strain-free, fully minimized, and relaxed without any bond, angle, or torsion angle restraints, and van der Waals atomic clashes. The final BanLec-eGFP structure comprised 384 amino acids, which encode for the BanLec, linker GSEF, and enhanced green fluorescent protein (eGFP). The strategy of cloning eGFP in the vector that already contains the BanLec gene sequence was conducted using NdeI (Thermo Fisher Scientific, Waltham, MA, USA) and BamHI (Thermo Fisher Scientific) restriction enzymes.

\subsection{Expression of BanLec-eGFP}

Escherichia coli BL21 (DE3)-pLysS (Agilent Technologies Inc., La Jolla, CA, USA) strain was employed as host for the expression of recombinant chimera. Bacteria were grown in LB medium containing $100 \mu \mathrm{g} / \mathrm{mL}$ of ampicillin and $25 \mu \mathrm{g} / \mathrm{mL}$ of chloramphenicol until optical density (OD) at $600 \mathrm{~nm}$ of $0.6-0.8$ was reached. The BanLec-eGFP synthesis was induced by adding isopropyl-D-thiogalactopyranoside (IPTG) (VWR International, LLC, Radnor, PA, USA) at a concentration of $1 \mathrm{mM}$, following incubation for 5 and $12 \mathrm{~h}$ at $25{ }^{\circ} \mathrm{C}$. After expression, the cell pellet obtained by centrifugation $(3000 \times g$ for $20 \mathrm{~min})$ was resuspended in $150 \mathrm{mM} \mathrm{NaCl}, 20 \mathrm{mM}$ tris(hydroxymethyl)aminomethane (TRIS) $\mathrm{HCl}$ $\mathrm{pH} 7.6$, and sonicated by the ultrasonic horn. The supernatant obtained by centrifugation $(3000 \times g$ for $20 \mathrm{~min}$ ) was used for downstream processing of the expressed chimera.

\subsection{Purification of Recombinant BanLec-eGFP}

BanLec-eGFP was purified by combining affinity chromatography on branched $\alpha$ 1,6 and $\alpha-1,3$ glucan polymer (Sephadex G-75 superfine, column volume $(\mathrm{CV})=10 \mathrm{~mL}$ ) and ion-exchange chromatography (HiTrap ANX column, $1 \mathrm{~mL}$, GE Healthcare, Little Chalfont, UK). After pre-equilibration with $20 \mathrm{mM}$ TRIS HCl pH 7.6, $150 \mathrm{mM} \mathrm{NaCl}$ (TRIS buffered saline, TBS), the matrix $(10 \mathrm{~mL})$ was incubated with the protein solution at room temperature for $1.5 \mathrm{~h}$. After the transfer to a column and thorough washing with five column volumes (CV) of TBS, bound BanLec-eGFP was eluted from the matrix with $0.3 \mathrm{M}$ glucose in TBS and collected in tubes $(1.5 \mathrm{~mL})$.

After affinity chromatography, collected samples were purified by ion-exchange chromatography connected to Äkta Purifier (GE Healthcare) using a combination of equilibration buffer (10 mM TRIS HCl, pH 8.0) and elution buffer (10 mM TRIS HCl, $0.5 \mathrm{M} \mathrm{NaCl}$, $\mathrm{pH} 8.0$ ), at room temperature and a flow rate of $1 \mathrm{~mL} / \mathrm{min}$. The column pressure limit was $0.3 \mathrm{MPa}$. Bound proteins were eluted using a linear gradient of elution buffer $(0-100 \%$ $(v / v)$, for 10 column volumes). The BanLec-eGFP was eluted at $50 \%$ of the elution buffer.

The wild type BanLec (GenBank accession number EU0556441) and BanLec H84T mutant were produced and purified according to the same protocol as BanLec-eGFP.

After purification, BanLec-eGFP protein was analyzed by Western blot. After blocking the membrane with 5\% skim milk in TBS with $0.05 \%$ Tween 20 (tTBS) for $1 \mathrm{~h}$, polyclonal rabbit anti-rBanLec antibodies [31] (dilution 1:10,000) were added, and the period of incubation was $1 \mathrm{~h}$. The membrane was washed, and alkaline phosphatase labeled goat anti-rabbit IgG (Sigma-Aldrich, St. Louise, MO, USA) was added. The binding of labeled antibodies for antigen was detected with nitro blue tetrazolium chloride/5-Bromo-4-chloro3-indolyl phosphate (Serva, Heidelberg, Germany).

\subsection{Analysis of the BanLec-eGFP Structure by CD Spectroscopy, Fluorescent and Mass Spectrometry}

For assessing the secondary structures of the recombinant BanLec-eGFP, Circular dichroism (CD) spectra were recorded at $25^{\circ} \mathrm{C}$ on Jasco J-815 circular dichroism spectropolarimeter (JASCO Inc., Easton, PA, USA) in the wavelength range from 185 to $260 \mathrm{~nm}$ in a cuvette with a path length of $0.1 \mathrm{~mm}$. The CD data were collected in intervals of $0.1 \mathrm{~nm}$, at a 
velocity of $50 \mathrm{~nm} / \mathrm{min}$. BanLec-eGFP was dialyzed against $20 \mathrm{mM}$ TRIS $\mathrm{HCl}$, pH 7.6 before spectral analysis. Purified BanLec-eGFP $(0.92 \mathrm{mg} / \mathrm{mL}, 80 \mu \mathrm{L})$ was used for the study.

The fluorescence of BanLec-eGFP was measured using a spectrofluorimeter FLUORO MAX-4 Jobin Yvon (HORIBA, Kyoto, Japan). The emission spectra were recorded between $400-650 \mathrm{~nm}$. The concentration of BanLec-eGFP used for the analysis was $0.92 \mathrm{mg} / \mathrm{mL}$ in 20 mM TRIS $\mathrm{HCl}$ buffer, pH 7.6.

To confirm the amino acid sequence, BanLec-eGFP was analyzed by mass spectrometry. The protein sample was separated by SDS-PAGE, followed by Coomassie Blue staining of the gel. The protein band corresponding to BanLec-eGFP was excised and destained by $100 \mathrm{mM} \mathrm{NH}_{4} \mathrm{HCO}_{3} / 50 \%$ acetonitrile $(\mathrm{ACN})$. The gel pieces were then reduced for $40 \mathrm{~min}$ by $20 \mathrm{mM}$ dithiothreitol and subsequently alkylated with $50 \mathrm{mM}$ iodoacetamide for $30 \mathrm{~min}$ in the dark. The samples were digested with sequencing grade trypsin according to the manufacturer's instructions (Promega, Mannheim, Germany). Before MS analysis, samples were cleaned over the C18 zip tip (Millipore, MA, USA). For MS analyses of the tryptic fragments, $\alpha$-cyano-4-hydroxycinnamic acid as a matrix was used. Tryptic maps were obtained on matrix assisted laser desorption ionization-time of flight/time of flight (MALDI-TOF/TOF) Ultraflextreme mass spectrometer (Bruker) operating in a reflectron positive mode and a peptide calibration standard mixture (Bruker 206195) was used for mass calibration.

The obtained peptide masses were searched against the NCBI protein sequence database using the MASCOT program (http:/ /www.matrixscience.com/search_form_ select.html).

\subsection{Competitive FLLSA (Fluorescence-Linked Lectin Sorbent Assay)}

Recombinant BanLec-eGFP chimera was employed in competitive FLLSA for the influenza virus detection to test its applicability in high-mannose binding assays. The assay was performed by inhibiting the BanLec-eGFP binding to the immobilized hemagglutinin from a Vaxigrip ${ }^{\mathrm{TM}}$ vaccine (Sanofi Pasteur, Lyon, France) with monosaccharides (mannose or glucose), wild type BanLec, and BanLec H84T mutant. Black 96-Well Immuno plates (Thermo Fisher Scientific) were coated with hemagglutinin ( $0.5 \mu \mathrm{g}$ per well) in $15 \mathrm{mM}$ $\mathrm{Na}_{2} \mathrm{CO}_{3} / 35 \mathrm{mM} \mathrm{NaHCO}$, pH 9.5 overnight at $4{ }^{\circ} \mathrm{C}$. Plates were washed with tPBS (phosphate buffered saline, PBS with $0.05 \%$ Tween 20). Then, the plates were blocked with $3 \%$ bovine serum albumin (BSA) in tPBS for $1 \mathrm{~h}$ at $37^{\circ} \mathrm{C}$. After washing, BanLec-eGFP (50 $\mu \mathrm{g}$ per well) was mixed without (control) or with different amounts of monosaccharides $(0.01,0.1,1,10,100,1000,5000 \mathrm{mM}$ ) or BanLec inhibitors (BanLec wt, or BanLec H84T; 0.01, $0.1,1$, and $10 \mu \mathrm{g}$ per well), which were added to the wells, and incubated in the dark for $2 \mathrm{~h}$ at room temperature. The FLLSA was performed in triplicate and the emission was measured at $535 \mathrm{~nm}$ on a fluorescent ELISA reader. Percent of inhibition was calculated as

$$
\% \text { of inhibition }=100 \times\left(1-\frac{(\mathrm{X}-\mathrm{min})}{(\max -\min )}\right)
$$

and IC50 value was determined as a concentration of inhibitor at 50\% of inhibition.

\subsection{Assessment of Fluorescence-Linked Lectin Binding to Bacterial Cells with Flow Cytometry}

For the assessment of recombinant BanLec-eGFP binding to bacterial cells, 7 strains of proteobacteria, species Salmonella enterica, subspecies enterica were used. The bacteria used were: serovar Enteritidis ATCC 13076; serovar Enteritidis clinical isolate E; serovar Typhimurium isolate B; serovar Typhimurium ATCC 14028; serovar Typhimurium isolate 2865; serovar Typhi clinical isolate 1243; and serovar Typhi clinical isolate 12 . The bacteria were cultivated in nutritious broth for $24 \mathrm{~h}$ and washed twice in PBS, by centrifugation at $2000 \times g$ for $15 \mathrm{~min}$. Cell density was adjusted to be the same for all strains, giving optical density of 0.15 at $620 \mathrm{~nm}$ in a $100 \mu \mathrm{L}$ volume in ELISA plate. The staining with BanLec-eGFP was done by aliquoting the bacteria and adding BanLec-eGFP. Incubation period was $15 \mathrm{~min}$, at $4{ }^{\circ} \mathrm{C}$, in the dark; followed by washing with PBS and centrifugation 
$2000 \times g$ for 15 min two times. Finally, the bacteria were resuspended in PBS with $0.4 \%$ formaldehyde (PBS-FA).

Inhibition with monosaccharides was performed on serovar Typhimurium isolate 2865. The bacterial suspensions were centrifuged for $15 \mathrm{~min} 2000 \times \mathrm{g}$ and PBS was removed. The bacteria were vortexed and different amounts of monosaccharides were added. This was followed by the addition of $1 \mu \mathrm{g}$ BanLec-eGFP. After incubation for $15 \mathrm{~min}$, at $4{ }^{\circ} \mathrm{C}$, in the dark, the bacteria were washed twice by adding PBS, centrifuged as before, and resuspended in PBS-FA. Each concentration point was done in quadruplicate.

The staining with BanLec-B (biotin labeled BanLec) was done the same way, except that after washing the unbound BanLec-B $0.2 \mu \mathrm{g}$ streptavidin-phycoerythrin (PE) (eBioscience) was added and after $15 \mathrm{~min}$ of incubation, the bacteria were washed as before.

The signal was analyzed with FACSVerse with BD FACSuite software (BD Biosciences). The graphs were drawn and results analyzed with OriginPro 8 and GraphPad Prism software.

\section{Results}

\subsection{Design, Cloning, Expression, and Purification of Recombinant BanLec-eGFP}

The model of BanLec-eGFP chimera designed in silico and fully optimized with the CHARMM22 force field [30] is depicted in Figure 1. Both carbohydrate-binding sites of BanLec are highlighted, according to [32], for further analysis and discussion. The binding site 1 consists of the GG loop (14-15), GKFLD loop (129-133) with additional S16 andV88 residues, while the binding site 2 includes the GG loop (59-60), GDVVD loop (34-38) with additional T61 and F131 residues, which interact with the bound glycans stabilizing the protein-receptor complex.

Enhanced green fluorescent protein (eGFP) is built up as the $\beta$-barrel tertiary structure with the barrel's fluorescent chromophore. The chromophore is generated by cyclization of T66, Y67, and G68 backbone atoms, and its central position is shown in Figure 1A. On the other hand, the $\beta$-strands with loops and turns define the tertiary structure of BanLec.

A

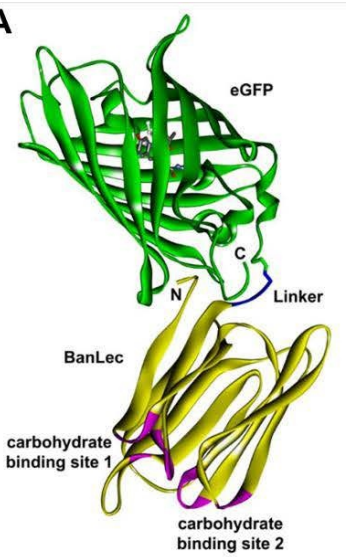

C

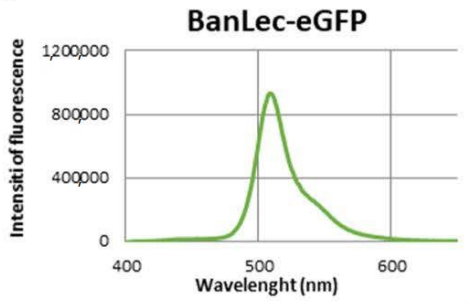

B

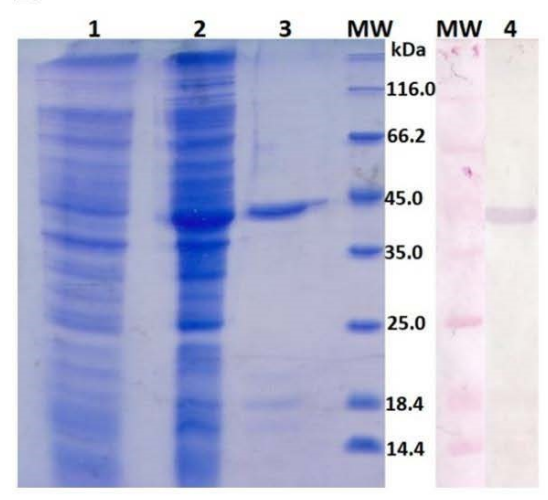

D

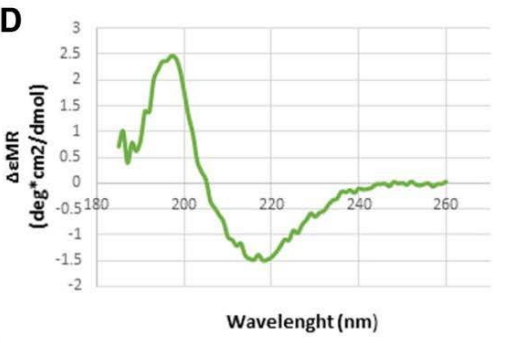

Figure 1. Cont. 


\section{E}
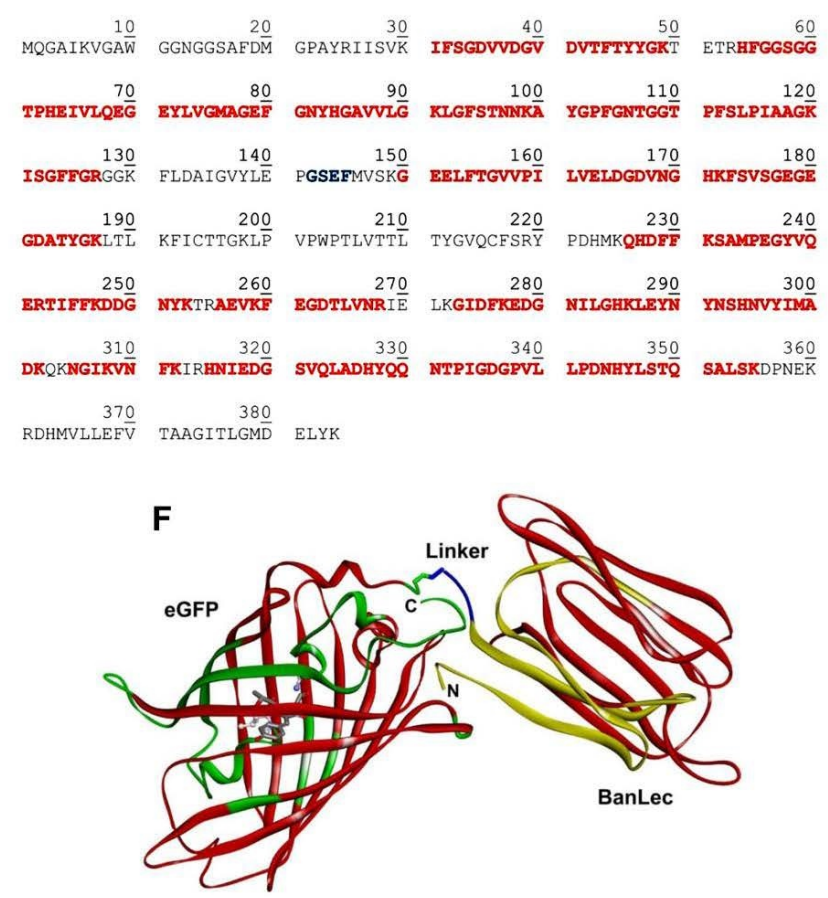

Figure 1. (A) The model structure of banana lectin-enhanced green fluorescent protein (BanLeceGFP) chimera is rendered as a solid ribbon. BanLec is shown in yellow, eGFP in green, glycineserine-glutamate-phenylalanine (GSEF) linker in blue and carbohydrate-binding sites in pink. The chromophore inside of eGFP is displayed as ball-and-stick. The depiction is made in Discovery Studio 19.1.0 [28]. (B) SDS-PAGE represents the profile of expression and purification of BanLec-eGFP (1-cell lysate before the addition of IPTG; 2-cell lysate after overnight expression; 3-BanLec-eGFP after purification; 4-detection of BanLec-eGFP by the polyclonal rabbit anti-rBanLec antibodies; MW, molecular weight marker). (C) Fluorescence spectra of BanLec-eGFP chimera. (D) Circular dichroism (CD) spectra of BanLec-eGFP in the far-ultraviolet region (185-260 nm). (E) The amino acid sequence of BanLec-eGFP chimera. The amino acid residues marked in red color represent the tryptic peptides that have been experimentally confirmed by the MS analysis. The linker GSEF is marked in blue. The theoretical $\mathrm{pI}=5.66$ and $\mathrm{Mw}=41,925.32 \mathrm{Da}$ for the BanLec-eGFP chimera. $(\mathbf{F})$ The model structure of the BanLec-eGFP construct was rendered in ribbon representation. The fragments of the structure experimentally confirmed are shown in red, while the other parts of BanLec, linker, and eGFP are displayed in yellow, blue, and green, respectively. The chromophore is shown in stick rendering in a gray color. The depiction was made in Discovery Studio [28].

BanLec-eGFP chimera structure appears to be stable over the time of simulation, mostly retaining the secondary and tertiary structure of both domains. The position of eGFP should not affect the binding capacity of BanLec for the glycans.

Gene for eGFP was cloned in the pET-23b-BL vector, and the resulting protein was expressed in E. coli BL21 (DE3)-pLysS. The addition of IPTG induced the expression of BanLec-eGFP. The produced recombinant protein was purified by combining affinity chromatography on a Sephadex G-75 superfine matrix and ion-exchange chromatography on an ANX column. The BanLec-eGFP chimeric protein was confirmed with Western blot using the polyclonal rabbit anti-rBanLec antibodies and alkaline phosphatase labeled anti-rabbit IgG antibodies (Figure 1B).

The yield of BanLec-eGFP after purification was about $16 \mathrm{mg}$ of protein per liter of cell culture. The purified protein showed a band of approximately $42 \mathrm{kDa}$ in SDS-PAGE under reducing conditions. The theoretical molecular mass of BanLec-eGFP calculated by the Compute pI/Mw tool (https:/ / web.expasy.org/compute_pi/) was 41,925.32 Da. 


\subsection{Physical-Chemical Characterization of BanLec-eGFP}

Physical-chemical characterization of BanLec-eGFP chimeric protein was performed by fluorescent spectroscopy, CD spectroscopy, and mass spectrometry. Fluorescent spectroscopy analysis revealed an emission maximum of BanLec-eGFP at $509 \mathrm{~nm}$ (Figure 1C).

Secondary structures of BanLec-eGFP were analyzed by CD spectroscopy in the far-UV region from 185-260 nm (Figure 1D). The protein's secondary structures were predicted by the K2D program (http://cbdm-01.zdv.uni-mainz.de/ andrade/k2d2/), which revealed the presence of $1.64 \%$ of $\alpha$-helix and $40.58 \%$ of $\beta$-strand in the BanLec-eGFP. The spectra of BanLec-eGFP showed well-defined secondary structures in the construct.

A peptide mass fingerprint was employed to confirm the primary structure of the BanLec-eGFP. MS spectrometry data were compared with theoretically obtained data using amino acid sequences (Source: https://web.expasy.org/peptide_cutter/). Peptides are shown in Table 1.

Table 1. Theoretical and peptide masses of the tryptic fragments obtained by MS analysis. AA = amino acid. M= molecular weight.

\begin{tabular}{|c|c|c|c|}
\hline [M] Determined & [M] Theoretical & Peptide Sequence & Peptide Length (-AA) \\
\hline 4474.01 & 4473.84 & HNIEDGSVQLADHYQQNTPIGDGPVLLPDNHYLSTQSALSK & 41 \\
\hline 3914.708 & 3915.35 & HFGGSGGTPHEIVLQEGEYLVGMAGEFGNYHGAVVLGK & 38 \\
\hline 2438.294 & 2437.73 & GEELFTGVVPILVELDGDVNGHK & 23 \\
\hline 2082.038 & 2082.29 & IFSGDVVDGVDVTFTYYGK & 19 \\
\hline 2023.074 & 2023.28 & AYGPFGNTGGTPFSLPIAAGK & 21 \\
\hline 1973.927 & 1974.18 & LEYNYNSHNVYIMADK & 16 \\
\hline 1542.819 & 1542.77 & GIDFKEDGNILGHK & 14 \\
\hline 1503.769 & 1503.54 & FSVSGEGEGDATYGK & 15 \\
\hline 1477.842 & 1477.75 & AEVKFEGDTLVNR & 13 \\
\hline 1347.748 & 1347.64 & TIFFKDDGNYK & 11 \\
\hline 1266.604 & 1266.39 & SAMPEGYVQER & 11 \\
\hline 1050.576 & 1050.14 & FEGDTLVNR & 9 \\
\hline 880.475 & 879.97 & LGFSTNNK & 8 \\
\hline 821.416 & 820.90 & QHDFFK & 6 \\
\hline 783.39 & 782.90 & ISGFFGR & 7 \\
\hline 655.336 & 654.81 & TIFFK & 5 \\
\hline 507.184 & 506.60 & VNFK & 4 \\
\hline 430.225 & 430.50 & NGIK & 4 \\
\hline
\end{tabular}

By mass analysis, more than $65 \%$ of the primary sequence was confirmed (Figure 1E). While only one amino acid V88 belonging to the binding site 1 is experimentally verified, all amino acid residues from the binding site 2 are confirmed by the MS analysis.

\subsection{Competitive Inhibition of BanLec-eGFP Binding to Influenza Vaccine High-Mannose Glycans}

BanLec-eGFP reactivity towards high-mannose glycans present on the influenza virus vaccine was assessed by competitive FLLSA inhibition with monosaccharides mannose and glucose, and wt BanLec and BanLec H84T. This assay was done with two controls, without hemagglutinin (min) and inhibitor (max). The IC50 value for wt BanLec was $9.12 \times 10^{-8} \mathrm{M}$, while IC50 for BanLec H84T was $2.35 \times 10^{-7} \mathrm{M}$, indicating 2.6 higher inhibition with wt BanLec in the employed experimental settings. In accordance with our previous findings, mannose was more efficient inhibitor for BanLec carbohydrate binding (Figure 2B) than glucose [11]. 

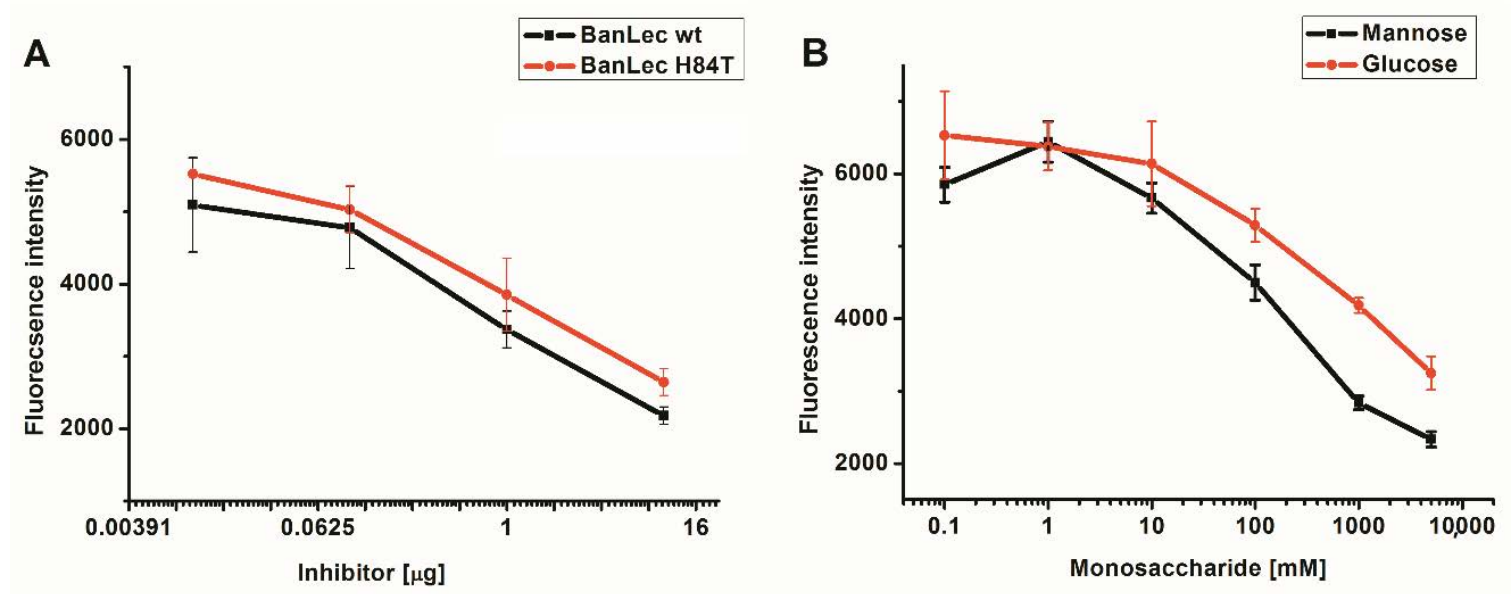

Figure 2. Inhibition of BanLec-eGFP binding to influenza vaccine high-mannose glycans with BanLec wt and BanLec H84T (A), and with monosaccharides (B), in fluorescence-linked lectin sorbent assay (FLLSA). The experiment was repeated three times in triplicate.

\subsection{Flow Cytometric Detection of BanLec-eGFP binding to bacteria}

The binding of BanLec-eGFP was detected to certain strains of Salmonella. As can be seen in Figure 3, the binding was not uniform and showed differences between the strains, with especially pronounced binding to serovar Typhi isolate 12 and serovar Typhimurium isolate 2865, Figure 3A,B. The binding was also detected to Salmonella enterica subsp. enterica serovar Typhimurium ATCC 14028 and serovar Typhi isolate 1243, Figure 3C,D. Essentially no binding was detected for the other strains tested, Figure $3 \mathrm{E}-\mathrm{G}$. The dependence of relative fluorescence (FITC-A Median) on the concentration of BanLec-eGFP used to stain different strains of Salmonella is shown in Figure $3 \mathrm{H}$.

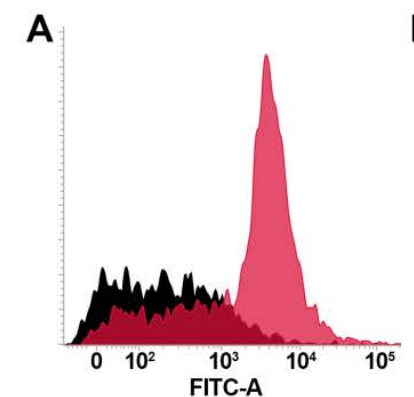

$\mathbf{E}$

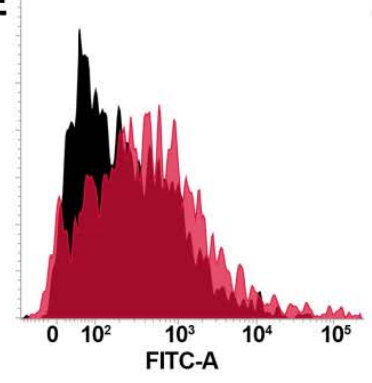

B

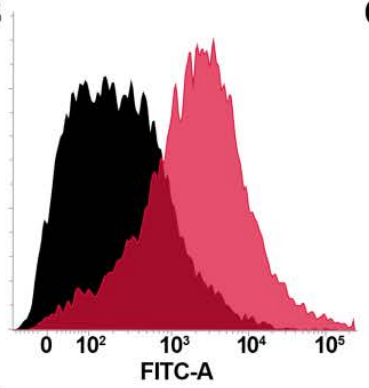

F

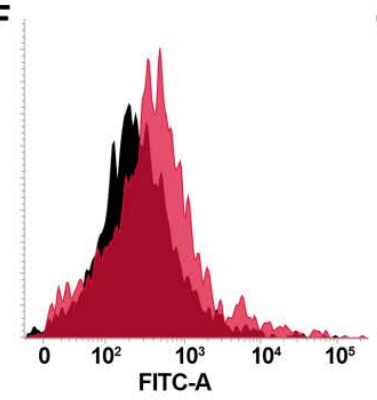

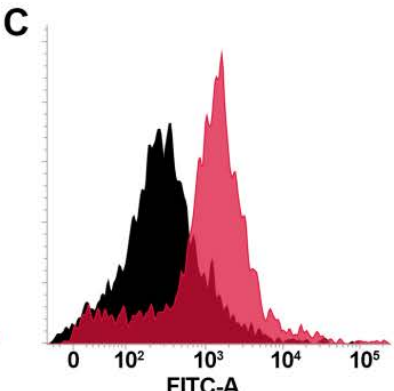

G

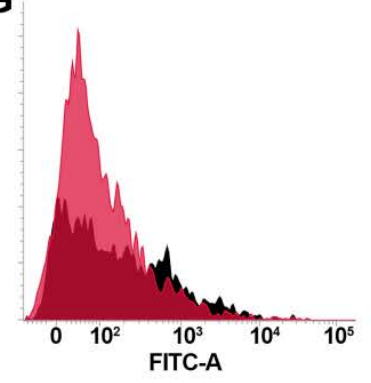

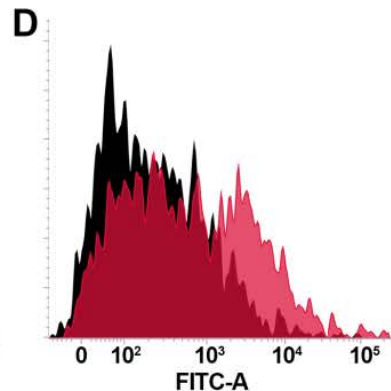

H

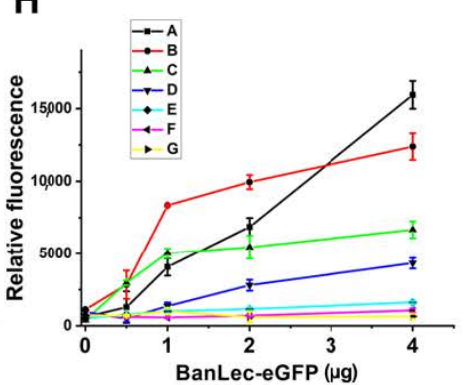

Figure 3. Flow cytometric analysis of the binding of BanLec-eGFP chimera to different strains of Salmonella enterica, subspecies enterica. (A) serovar Typhi clinical isolate 12; (B) serovar Typhimurium isolate 2865; (C) serovar Typhimurium ATCC 14028; (D) serovar Typhi clinical isolate 1243; (E) serovar Typhimurium isolate B; (F) serovar Enteritidis clinical isolate E; (G) serovar Enteritidis ATCC 13076; Black histogram—unstained bacteria; pink histogram—bacteria stained with $14 \mu \mathrm{g}$ of BanLec-eGFP chimera; (H) dependence of FITC-A Median, labeled relative fluorescence on the concentration of BanLec-eGFP chimera. The strains are labeled as described above. 
The binding of BanLec-eGFP was completely dependent on lectin-carbohydrate interaction as complete inhibition could be achieved with both mannose and glucose, Figure 4A,B. Galactose could not inhibit this interaction even at a high concentration of $0.1 \mathrm{M}$. Inhibition with mannose gave IC50 $\leq 1.7 \mathrm{mM}$, which was substantially lower than that obtained with glucose IC50 $=7.4 \mathrm{mM}$, Figure $4 \mathrm{~B}$.
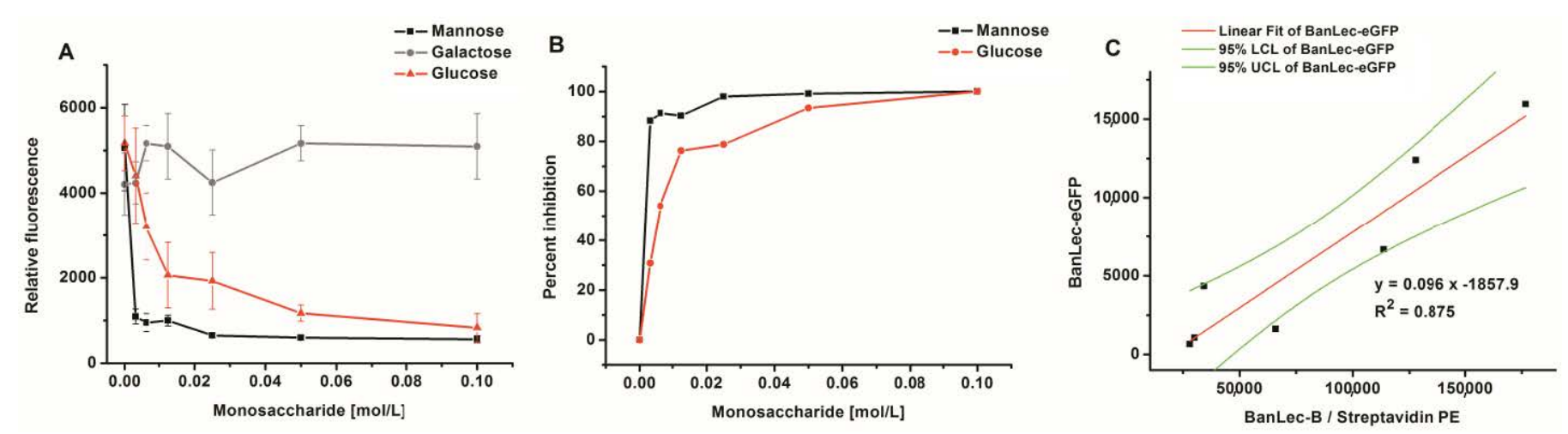

Figure 4. Inhibition of BanLec-eGFP binding to Salmonella enterica, subspecies enterica serovar Typhimurium isolate 2865 with different amounts of monosaccharides; panels $(\mathbf{A}, \mathbf{B})$ and correlation of staining with BanLec-eGFP with the staining with BanLec-biotin (BanLec-B) and streptavidin-phycoerythrin (PE)—panel (C), using flow cytometry. Each point was run in quadruplicate. Panel: (A) relative fluorescence; (B) percentage of inhibition; (C) correlation. For correlation experiments, $4 \mu \mathrm{g}$ of BanLec-eGFP was used and $0.5 \mu \mathrm{g}$ of BanLec-B with $0.2 \mu \mathrm{g}$ streptavidin-PE (eBioscience) was used. LCL, lower confidence limit; UCL, upper confidence limit.

In order to compare the binding of BanLec-eGFP to Salmonella enterica serovar Typhimurium isolate 2865 to that which would be obtained by using BanLec-B and streptavidin$\mathrm{PE}$, a correlation was made, Figure 4C. A correlation coefficient of 0.875 was obtained between the two staining procedures, with very little optimization, showing that the correlation was highly significant $(p=0.0019)$.

\section{Discussion}

In this study, recombinant BanLec-eGFP chimera was in silico designed, produced, and tested for application in the binding assay for high mannose structures present on microorganisms.

The chimera retained the secondary structures of both individual proteins and the structure was stable over the time of simulation, mostly retaining the secondary and tertiary structures of both domains, and further on as demonstrated by functional assays. The position of eGFP did not affect the binding capacity of BanLec for the glycans. This was evaluated by testing the binding of BanLec-eGFP chimera to influenza virus antigens and to different strains of Salmonella. These microorganisms were selected for analysis because of the presence of mannose on their surface and because of the medical relevancy.

Viruses utilize envelope proteins for receptor recognition at the entry point into the host cell. The antiviral activity of lectins is based on binding to oligosaccharides attached to the viral envelope glycoproteins and inhibiting viral fusion and entry [33].

Many viruses such as HIV, severe acute respiratory syndrome corona viruses (SARS$\mathrm{CoV}$, SARS-CoV-2), herpes simplex virus (HSV), Ebola, hepatitis B virus (HBV), hepatitis C virus (HCV), influenza, West Nile virus, dengue virus, and zika virus have glycosylated envelope proteins $[33,34]$. The importance of protein-sugar interactions is best illustrated by the fact that the currently used therapeutics against influenza, Tamiflu (Oseltamivir) and Relenza (Zanamivir), function as sialic acid analogs [35].

Antiviral lectins interact with glycan structures added as post-translational modifications to the viral envelope proteins [34,36]. The anti-HIV activity has been reported for the mannose-binding lectins such as the lectin from banana (BanLec), microvirin from the cyanobacterium Microcystis aeruginosa, scytovirin from the cyanobacteria Scytonema varium, 
lectin from snowdrop (GNA), and lectin from the red alga Griffithsia sp. (GriffithsinGRFT). They have been proposed as antiviral microbicides in the prevention of HIV transmission [36,37]. Anti-influenza activity has been shown for some lectins, such as BanLec [38], cyanovirin-N [39], NICTABA (Nicotiana tabacum agglutinin), and UDA (Urtica dioica agglutinin) [40].

Various lectins have been fuzzed with fluorescent proteins as fluorescent probes for the detection of specific glycan structures present on the cell-surface or intracellular conjugates, making them versatile primary detection reagents. The neurocan-GFP was successfully employed for the detection of hyaluronan tissue distribution [24]. Fluorescently labeled Aspergillus oryzae lectin was a useful probe for the detection of the core fucose in N-glycans, but can be also useful vehicles for delivery of substances into the cells [26].

Vaxigrip ${ }^{\circledR}$, Sanofi Pasteur IIV3, used in this study is a trivalent vaccine, containing antigens from A/H1N1, A/H3N2, and B strains, inactivated A and B split-virion influenza vaccine, (15 $\mu \mathrm{g}$ of haemagglutinin for each strain) [41]. By employing BanLec-eGFP in the competitive FLLSA, it was shown that wt BanLec exhibits higher binding for high mannose glycans present on the employed influenza vaccine than the H84T BanLec mutant, contrary to some literature data $[14,38]$.

In order to assess the binding potential of BanLec-eGFP to highly mannosylated microorganisms, based on our experimental data and literature data, we decided to use Sallmonella. Previously we demonstrated that selectivity of rBanLec to fungal $\beta$-glucans can be achieved in enzyme linked lectin sorbent assay (ELLSA) [42]. The ability of BanLec to bind fungal $\beta$-glucans has been demonstrated previously by Goldstein et al. [43]. On that occasion [42] Salmonella was not included in the analysis. After conducting ELLSA to test the binding of BanLec to Salmonella, we detected approximately equal binding to that obtained with yeast cells.

Salmonella is a well-known bacterial pathogen that causes both food-borne gastroenteritis in humans and animals and typhoid fever. There are currently two species of Salmonella, with Salmonella enterica being medically relevant. Within the species of $S$. enterica individual subspecies and serovars have been identified. Different serovars are differentiated by the structures of their flagellum, carbohydrates, and lipopolysaccharides (LPS) [44,45]. Certain strains of Salmonella are known to contain mannose-rich LPS, and the binding of Mannosebinding lectin (MBL) to this LPS has been investigated [46-48]. Studies on the effect of MBL binding to $S$. enterica serovar Typhimurium have revealed the bactericidal properties of MBL $[39,40]$. The inhibitory effect of MBL on the motility of Salmonella occurs by affecting the energy source required for motility and the signaling pathway of chemotaxis [48].

The binding of MBL to a strain of Salmonella producing a mannose-rich LPS was previously demonstrated with the similar principle employed in this study, by the binding of fluorochrome conjugated anti-MBL serum [46]. The approach used in this study is a direct one, with the novel BanLec-eGFP chimera, and the results indicate that BanLec-eGFP acts in a similar fashion as MBL, which remains to be corroborated by parallel testing.

Important to note is that we have obtained differences between individual serovars and strains. In particular, serovar Typhi clinical isolate 12, showed highest binding. The tested strains from serovar Enteritidis showed no binding, while variations between strains in the serovar Typhimurium have been noticed. MBL does not bind to nonpathogenic mutants of Salmonella enterica serovar Montevideo that lack the mannose-rich O-polysaccharide within LPS [46] leading to the question whether mannosylation influences the pathogenicity of these organisms and in what way. The novel BanLec-eGFP chimera represents an excellent novel tool for such analysis.

In this study, we have demonstrated by inhibition experiments with monosaccharides that the binding of BanLec-eGFP to microorganisms occurs through the interaction of the lectin with the saccharide component. Moreover, we have demonstrated that there is no significant influence of the addition of GFP to the specificity of BanLec.

Each lectin molecule is characterized by affinity, specificity, and selectivity, and the comparison of different lectins can be done within basic research studies. We previously 
compared BanLec with other mannose binding lectins such as Concanavalin A and Lens culinaris agglutinin (LCA) and found profound differences [42]. In biotechnology, often relying on the knowledge obtained through basic research, a more targeted approach, with a specific purpose should always be applied. Based on the results presented above and other literature data, further studies including the comparison of MBL with BanLec should be performed.

In conclusion, BanLec-eGFP was produced in high yield, with retained secondary structures in both domains. The protein is stable and offers the advantage of constant emission capacity, unlike the chemically labeled lectin molecules, which increases reproducibility.

Author Contributions: Conceptualization, M.G.-J. and R.M.; Methodology, Z.L., L.D., U.A., D.P., Software, D.P.; Validation, M.G.-J. and D.P.; Formal Analysis, Z.L.; Investigation, Z.L., L.D.; Resources, M.G.-J.; Writing-Original Draft Preparation, Z.L., D.P., and M.G.-J.; Writing-Review \& Editing, M.G.-J.; Visualization, R.M.; Supervision, M.G.-J.; L.D.; Funding Acquisition, M.G.-J. All authors have read and agreed to the published version of the manuscript.

Funding: This work was financially supported by the Ministry of Education, Science and Technological Development of the Republic of Serbia (Contract number: 451-03-68/2020-14/200168, Contract number: 451-03-68/2020-14/200177, and Contract number: 451-03-68/2020-14/200026).

Institutional Review Board Statement: Not applicable.

Informed Consent Statement: Not applicable.

Data Availability Statement: The data that support the findings of this study are available from the corresponding author M.G.-J., upon reasonable request.

Acknowledgments: This paper was supported by bilateral scientific project financed by Ministries of Sciences of Croatia and Serbia.

Conflicts of Interest: The authors declare no conflict of interest.

\section{References}

1. Lam, S.-K.; Ng, T. Lectins: Production and practical applications. Appl. Microbiol. Biotechnol. 2010, 89, 45-55. [CrossRef]

2. Sharon, N.; Lis, H. Lectins as cell recognition molecules. Science 1989, 246, 227-234. [CrossRef]

3. Jain, P.; Bhuiyan, M.H.; Hossain, K.R.; Bachar, S.C. Antibacterial and antioxidant activities of local seeded banana fruits. Afr. J. Pharm. Pharmacol. 2011, 5, 1398-1403. [CrossRef]

4. Koshte, V.L.; Van Dijk, W.; Van Der Stelt, M.E.; Aalberse, R.C. Isolation and characterization of BanLec-I, a mannoside-binding lectin from Musa paradisiac (banana). Biochem. J. 1990, 272, 721-726. [CrossRef]

5. Peumans, W.J.; Zhang, W.; Barre, A.; Astoul, C.H.; Balint-Kurti, P.J.; Rovira, P.; Rougé, P.; May, G.D.; Van Leuven, F.; Truffa-Bachi, P.; et al. Fruit-specific lectins from banana and plantain. Planta 2000, 211, 546-554. [CrossRef]

6. Singh, D.D.; Saikrishnan, K.; Kumar, P.; Dauter, Z.; Sekar, K.; Surolia, A.; Vijayan, M. Purification, crystallization and preliminary X-ray structure analysis of the banana lectin fromMusa paradisiaca. Acta Crystallogr. Sect. D Biol. Crystallogr. 2004, 60, 2104-2106. [CrossRef]

7. Khan, J.M.; Qadeer, A.; Ahmad, E.; Ashraf, R.; Bhushan, B.; Chaturvedi, S.K.; Rabbani, G.; Khan, R.H. Monomeric Banana Lectin at Acidic pH Overrules Conformational Stability of Its Native Dimeric Form. PLoS ONE 2013, 8, 1-12. [CrossRef]

8. Koshte, V.; Aalbers, M.; Calkhoven, P.; Aalberse, R. The Potent lgG4-lnducing Antigen in Banana Is a Mannose-Binding Lectin, BanLec-I. Int. Arch. Allergy Immunol. 1992, 97, 17-24. [CrossRef]

9. Swanson, M.D.; Winter, H.C.; Goldstein, I.J.; Markovitz, D.M. A Lectin Isolated from Bananas Is a Potent Inhibitor of HIV Replication. J. Biol. Chem. 2010, 285, 8646-8655. [CrossRef]

10. Hopper, J.T.; Ambrose, S.; Grant, O.C.; Krumm, S.A.; Allison, T.M.; Degiacomi, M.T.; Tully, M.D.; Pritchard, L.K.; Ozorowski, G.; Ward, A.B.; et al. The Tetrameric Plant Lectin BanLec Neutralizes HIV through Bidentate Binding to Specific Viral Glycans. Structure 2017, 25, 773-782. [CrossRef]

11. Gavrovic-Jankulovic, M.; Poulsen, K.; Brckalo, T.; Bobic, S.; Lindner, B.; Petersen, A. A novel recombinantly produced banana lectin isoform is a valuable tool for glycoproteomics and a potent modulator of the proliferation response in CD3+, CD4+, and CD8+ populations of human PBMCs. Int. J. Biochem. Cell Biol. 2008, 40, 929-941. [CrossRef] [PubMed]

12. Stojanovic, M.; Zivkovic, I.; Petrušić, V.; Kosec, D.J.; Dimitrijević, R.D.; Jankov, R.M.; Dimitrijević, L.A.; Gavrovic-Jankulovic, M. In vitro stimulation of Balb/c and C57 BL/6 splenocytes by a recombinantly produced banana lectin isoform results in both a proliferation of $\mathrm{T}$ cells and an increased secretion of interferon-gamma. Int. Immunopharmacol. 2010, 10, 120-129. [CrossRef] [PubMed] 
13. Dimitrijević, R.; Stojanovic, M.; Micic, M.; Gavrovic-Jankulovic, M. Recombinant banana lectin as mucosal immunostimulator. J. Funct. Foods 2012, 4, 636-641. [CrossRef]

14. Swanson, M.D.; Boudreaux, D.M.; Salmon, L.; Chugh, J.; Winter, H.C.; Meagher, J.L.; André, S.; Murphy, P.V.; Oscarson, S.; Roy, R.; et al. Engineering a Therapeutic Lectin by Uncoupling Mitogenicity from Antiviral Activity. Cell 2015, 163, 746-758. [CrossRef]

15. Brawek, B.; del Moral, M.O.; Garaschuk, O. In Vivo Visualization of Microglia Using Tomato Lectin. In Methods in Molecular Biology; Humana Press: New York, NY, USA, 2019.

16. Liao, W.-Y.; Fugmann, S.D. Lectins identify distinct populations of coelomocytes in Strongylocentrotus purpuratus. PLoS ONE 2017, 12, 1-21. [CrossRef]

17. Lohr, M.; Kaltner, H.; Schwartz-Albiez, R.; Sinowatz, F.; Gabius, H.-J. Towards Functional Glycomics by Lectin Histochemistry: Strategic Probe Selection to Monitor Core and Branch-end Substitutions and Detection of Cell-type and Regional Selectivity in Adult Mouse Testis and Epididymis. Anat. Histol. Embryol. 2010, 39, 481-493. [CrossRef]

18. Wolters-Eisfeld, G.; Schumacher, U. Lectin Histochemistry for Metastasizing and Non-metastasizing Cancer Cells. In Histochemestry of Single Molecules; Humana Press: New York, NY, USA, 2017; Volume 1560, pp. 121-132. [CrossRef]

19. Neves, A.A.; Di Pietro, M.; O’Donovan, M.; Waterhouse, D.J.; Bohndiek, S.E.; Brindle, K.M.; Fitzgerald, R.C. Detection of early neoplasia in Barrett's esophagus using lectin-based near-infrared imaging: An ex vivo study on human tissue. Endoscopy 2018, 50, 618-625. [CrossRef]

20. Carvalho, M.E.T.; De Oliveira, W.F.; Cunha, C.R.; Coelho, L.C.; Silva, M.V.; Junior, L.B.C.; Santos, B.S.; Filho, P.E.C.; Fontes, A.; Correia, M.T. Evaluating the glycophenotype on breast cancer tissues with quantum dots-Cramoll lectin conjugates. Int. J. Biol. Macromol. 2019, 138, 302-308. [CrossRef]

21. Stevens, J.; Blixt, O.; Paulson, J.C.; Wilson, I.A. Glycan microarray technologies: Tools to survey host specificity of influenza viruses. Nat. Rev. Genet. 2006, 4, 857-864. [CrossRef]

22. Fei, Y.; Sun, Y.-S.; Li, Y.; Lau, K.; Yu, H.; Chokhawala, H.A.; Huang, S.; Landry, J.P.; Chen, X.; Zhu, X. Fluorescent labeling agents change binding profiles of glycan-binding proteins. Mol. BioSyst. 2011, 7, 3343-3352. [CrossRef]

23. Giepmans, B.N.; Adams, S.R.; Ellisman, M.H.; Tsien, R.Y. The fluorescent toolbox for assessing protein location and function. Science 2006, 312, 217-224. [CrossRef] [PubMed]

24. Zhang, H.; Baader, S.L.; Sixt, M.; Kappler, J.; Rauch, U. Neurocan-GFP Fusion Protein. J. Histochem. Cytochem. 2004, 52, 915-922. [CrossRef]

25. Lannoo, N.; Peumans, W.J.; Van Pamel, E.; Alvarez, R.; Xiong, T.-C.; Hause, G.; Mazars, C.; Van Damme, E.J.M. Localization and in vitro binding studies suggest that the cytoplasmic/nuclear tobacco lectin can interact in situ with high-mannose and complexN-glycans. FEBS Lett. 2006, 580, 6329-6337. [CrossRef] [PubMed]

26. Mun, J.-Y.; Lee, K.J.; Kim, Y.J.; Kwon, O.; Lee, S.-G.; Park, W.S.; Heo, W.D.; Oh, D.-B. Development of fluorescent probes for the detection of fucosylated N-glycans using an Aspergillus oryzae lectin. Appl. Microbiol. Biotechnol. 2012, 93, 251-260. [CrossRef] [PubMed]

27. Arpino, J.A.J.; Rizkallah, P.J.; Jones, D.D. Crystal Structure of Enhanced Green Fluorescent Protein to $1.35 \AA ̊$ Resolution Reveals Alternative Conformations for Glu222. PLoS ONE 2012, 7, 1-8. [CrossRef] [PubMed]

28. Discovery Studio Modeling Environment, Release 4.5; BIOVIA, Dassault Systèmes: San Diego, CA, USA, 2015.

29. Phillips, J.C.; Braun, R.; Wang, W.; Gumbart, J.; Tajkhorshid, E.; Villa, E.; Chipot, C.; Skeel, R.D.; Kalé, L.; Schulten, K. Scalable molecular dynamics with NAMD. J. Comput. Chem. 2005, 26, 1781-1802. [CrossRef] [PubMed]

30. MacKerell, A.D.; Bashford, D.; Bellott, M.; Dunbrack, R.L.; Evanseck, J.D.; Field, M.J.; Fischer, S.; Gao, J.; Guo, H.; Ha, S.; et al. All-Atom Empirical Potential for Molecular Modeling and Dynamics Studies of Proteins. J. Phys. Chem. B 1998, 102, $3586-3616$. [CrossRef] [PubMed]

31. Dimitrijevic, R.; Jadranin, M.; Burazer, L.; Ostojic, S.; Gavrovic-Jankulovic, M. Evaluation of the thermal stability and digestibility of heterologously produced banana lectin. Food Chem. 2010, 120, 1113-1118. [CrossRef]

32. Meagher, J.L.; Winter, H.C.; Ezell, P.; Goldstein, I.J.; Stuckey, J.A. Crystal structure of banana lectin reveals a novel second sugar binding site. Glycobiology 2005, 15, 1033-1042. [CrossRef]

33. Walls, A.C.; Park, Y.-J.; Tortorici, M.A.; Wall, A.; McGuire, A.T.; Veesler, D. Structure, Function, and Antigenicity of the SARS-CoV-2 Spike Glycoprotein. Cell 2020, 181, 281-292. [CrossRef]

34. Mitchell, C.A.; Ramessar, K.; O'Keefe, B.R. Antiviral lectins: Selective inhibitors of viral entry. Antivir. Res. 2017, 142, 37-54. [CrossRef] [PubMed]

35. Kim, J.-H.; Resende, R.; Wennekes, T.; Chen, H.-M.; Bance, N.; Buchini, S.; Watts, A.G.; Pilling, P.; Streltsov, V.A.; Petric, M.; et al. Mechanism-Based Covalent Neuraminidase Inhibitors with Broad-Spectrum Influenza Antiviral Activity. Science 2013, $340,71-75$. [CrossRef] [PubMed]

36. Zhou, R.; Wang, X.; Liu, H.; Guo, L.; Su, Q.; Wang, H.; Vasiliadis, T.; Ho, W.; Li, J. GalNAc-Specific Soybean Lectin Inhibits HIV Infection of Macrophages through Induction of Antiviral Factors. J. Virol. 2017, 92, 1-11. [CrossRef] [PubMed]

37. Koharudin, L.M.; Gronenborn, A.M. Antiviral lectins as potential HIV microbicides. Curr. Opin. Virol. 2014, 7, 95-100. [CrossRef]

38. Covés-Datson, E.M.; King, S.R.; Legendre, M.; Gupta, A.; Chan, S.M.; Gitlin, E.; Kulkarni, V.V.; García, J.P.; Smee, D.F.; Lipka, E.; et al. A molecularly engineered antiviral banana lectin inhibits fusion and is efficacious against influenza virus infection in vivo. Proc. Natl. Acad. Sci. USA 2020, 117, 2122-2132. [CrossRef] 
39. O'Keefe, B.R.; Smee, D.F.; Turpin, J.A.; Saucedo, C.J.; Gustafson, K.R.; Mori, T.; Blakeslee, D.; Buckheit, R.; Boyd, M.R. Potent Anti-Influenza Activity of Cyanovirin-N and Interactions with Viral Hemagglutinin. Antimicrob. Agents Chemother. 2003, 47, 2518-2525. [CrossRef]

40. Gordts, S.C.; Renders, M.; Férir, G.; Huskens, D.; Van Damme, E.J.M.; Peumans, W.; Balzarini, J.; Schols, D. NICTABA and UDA, two GlcNAc-binding lectins with unique antiviral activity profiles. J. Antimicrob. Chemother. 2015, 70, 1674-1685. [CrossRef]

41. Haugh, M.; Gresset-Bourgeois, V.; Macabeo, B.; Woods, A.; Samson, S.I. A trivalent, inactivated influenza vaccine (Vaxigrip ${ }^{\circledR}$ ): Summary of almost 50 years of experience and more than 1.8 billion doses distributed in over 120 countries. Expert Rev. Vaccines 2017, 16, 545-564. [CrossRef]

42. Dragacevic, L.; Djordjevic, B.; Gavrovic-Jankulovic, M.; Ilic, V.; Kanazir, D.; Minić, R. ELLSA based profiling of surface glycosylation in microorganisms reveals that ß-glucan rich yeasts' surfaces are selectively recognized with recombinant banana lectin. Glycoconj. J. 2019, 37, 95-105. [CrossRef]

43. Goldstein, I.J.; Winter, H.C.; Mo, H.; Misaki, A.; Van Damme, E.J.M.; Peumans, W.J. Carbohydrate binding properties of banana (Musa acuminata ) lectin. JBIC J. Biol. Inorg. Chem. 2001, 268, 2616-2619. [CrossRef]

44. Groisman, E.A.; Sturmoski, M.A.; Solomon, F.R.; Lin, R.; Ochman, H. Molecular, functional, and evolutionary analysis of sequences specific to Salmonella. Proc. Natl. Acad. Sci. USA 1993, 90, 1033-1037. [CrossRef] [PubMed]

45. Coburn, B.; Grassl, G.A.; Finlay, B.B. Salmonella, the host and disease: A brief review. Immunol. Cell Biol. 2007, 85, 112-118. [CrossRef] [PubMed]

46. Kuhlman, M.; Joiner, K.; Ezekowitz, R.A. The human mannose-binding protein functions as an opsonin. J. Exp. Med. 1989, 169, 1733-1745. [CrossRef] [PubMed]

47. Devyatyarova-Johnson, M.; Rees, I.H.; Robertson, B.D.; Turner, M.W.; Klein, N.J.; Jack, D.L. The Lipopolysaccharide Structures of Salmonella enterica Serovar Typhimurium and Neisseria gonorrhoeaeDetermine the Attachment of Human Mannose-Binding Lectin to Intact Organisms. Infect. Immun. 2000, 68, 3894-3899. [CrossRef] [PubMed]

48. Xu, J.; Nakamura, S.; Islam, S.; Guo, Y.; Ihara, K.; Tomioka, R.; Masuda, M.; Yoneyama, H.; Isogai, E. Mannose-Binding Lectin Inhibits the Motility of Pathogenic Salmonella by Affecting the Driving Forces of Motility and the Chemotactic Response. PLoS ONE 2016, 11, 1-14. [CrossRef] 